\title{
The FREEWAT platform for the assessment of water availability and quality
}

\section{La piattaforma FREEWAT per l'analisi della disponibilità idrica e della qualità delle acque}

Giovanna De Filippis - Istituto di Scienze della Vita, Scuola Superiore Sant’Anna, Pisa, Italy - g.defilippis@santannapisa.it

Iacopo Borsi - TEA SISTEMI S.p.A., Pisa, Italy - iacopo.borsi@tea-group.com

Matteo Ghetta - Istituto di Scienze della Vita, Scuola Superiore Sant'Anna, Pisa - matteo.ghetta@gmail.com

Rudy Rossetto - Istituto di Scienze della Vita, Scuola Superiore Sant’Anna, Pisa - r.rossetto@santannapisa.it

Keywords: FREEWAT, GIS, integrated hydrological model, groundwater management.

Parole chiave: FREEWAT, GIS, modello idrologico integrato, gestione delle acque sotterranee.

La piattaforma di simulazione FREEWAT (Rossetto et al. 2015; De Filippis et al. 2017) si propone agli utilizzatori e ai gestori della risorsa idrica sotterranea come uno strumento composito a supporto della tutela e della preservazione di un bene comune, di cui ormai molto spesso si parla in relazione ad eventi climatici estremi o ad attività antropiche che modificano profondamente l'equilibrio del sistema idro(geo)logico e degli ecosistemi annessi. Si tratta di un software libero e gratuito che permette di studiare svariate problematiche legate alla disponibilità della risorsa idrica e alla qualità delle acque, avvalendosi dell'integrazione di strumenti dedicati per l'analisi di dati e di codici robusti, distribuiti e fisicamente basati, in un ambiente di simulazione GIS, in cui i dati spaziali possono essere agevolmente processati, gestiti e visualizzati.

In tema di gestione della risorsa idrica sotterranea, FREEWAT integra strumenti che permettono di ottimizzare l'uso congiunto delle acque superficiali e sotterranee: si tratta del codice di simulazione MODFLOW-OWHM (One-Water Hydrologic flow Model; Hanson et al. 2014). Tale codice permette di rappresentare il flusso idrico tridimensionale in sistemi acquiferi porosi ed eterogenei, in zona satura e insatura, inclusi gli scambi con i corpi idrici superficiali. In MODFLOWOWHM, l'intero ciclo idrologico viene simulato implementando il modulo Farm Process (Schmid et al. 2006; Schmid and Hanson 2009; Hanson et al. 2014), grazie al quale è possibile accoppiare il bilancio idrico sotterraneo con quello di specifiche sotto-regioni del modello (chiamate farm o, in FREEWAT, water unit), per le quali viene calcolata una domanda idrica legata all'irrigazione di colture o al soddisfacimento di attività antropiche, minimizzando potenziali impatti negativi sulla quantità e sulla qualità della risorsa idrica sotterranea.

I termini del bilancio idrico calcolato per ogni farm sono legati sia alla domanda idrica della farm stessa che alla disponibilità di risorsa idrica (superficiale o sotterranea). È possibile infatti simulare le seguenti componenti (Fig. 1): traspirazione della pianta, evaporazione dal suolo, infiltrazione meteorica effettiva, disponibilità di acqua da corpi idrici superficiali e da falda, deflusso superficiale, percolazione dell'acqua in eccesso non assorbita dall'apparato radicale. Tutte queste componenti vengono integrate in maniera dinamica in base ad un ordine di priorità: la prima fonte di approvvigionamento per il soddisfacimento del fabbisogno idrico è data dalle fonti "naturali"

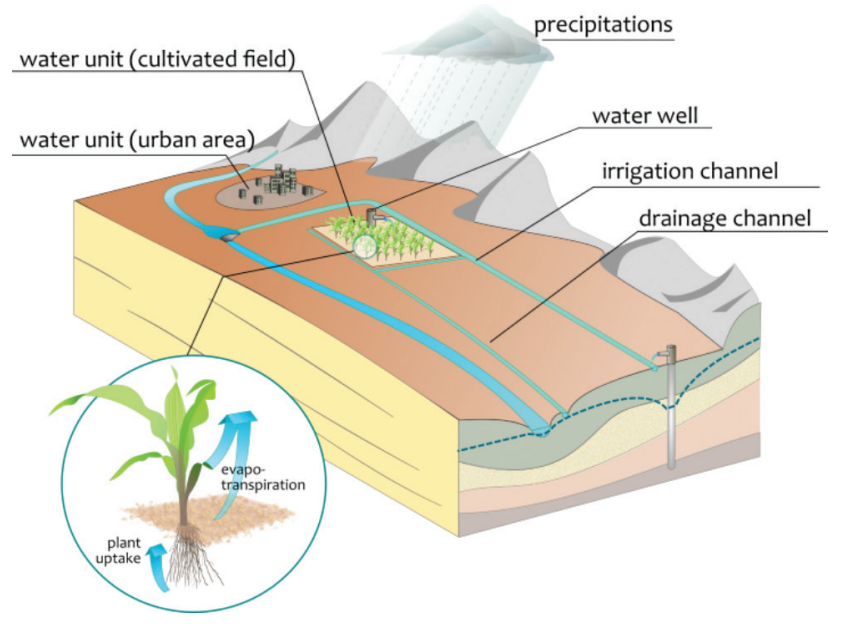

Fig.1: Schematizzazione delle componenti del ciclo idrologico che possono essere rappresentate in un'applicazione del Farm Process.

Fig. 1: Schematic representation of the main components of the bydrologic cycle, which can be simulated through the application of the Farm Process.

(infiltrazione di acqua meteorica e conseguente assorbimento radicale); nel caso in cui ciò non fosse sufficiente per soddisfare la richiesta idrica della farm, viene determinata una domanda residua e vengono individuate ulteriori fonti di approvvigionamento, a cui si attinge dando la priorità a quelle superficiali (se disponibili) e, solo in ultima istanza, a quelle sotterranee. Possono anche essere definiti dei vincoli sulla disponibilità idrica sia da corpi superficiali che da falda.

Il Farm Process permette dunque di implementare un modello integrato, il cui principale risultato è un bilancio completo per ognuna delle farm definite nel modello e implicitamente connesso a quello del sistema acquifero, in quanto la traspirazione delle colture e l'evaporazione dal suolo sono determinate in base al livello della falda superficiale. Tutte le componenti del suddetto bilancio idrico possono essere analizzate mediante uno strumento di post-processing integrato in FREEWAT, che permette di visualizzarle in un unico grafico e di rappresentarne la variabilità nel tempo. In questo modo è possibile anche identificare eventuali periodi di deficit, durante i quali la domanda di una determinata farm non può essere completamente soddisfatta dalla disponibilità idrica superficiale e/o sotterranea. In tal caso sono disponibili due metodi 
di ottimizzazione: il primo consiste nell'assegnare un ordine di priorità alle colture maggiormente idro-esigenti, mentre il secondo è basato sulla resa economica delle colture in relazione ai costi irrigui.

La piattaforma FREEWAT integra anche diversi codici per la simulazione del trasporto dei contaminanti in falda. Lapplicazione di tali strumenti consente di affrontare svariate problematiche in contesti ambientali anche molto diversi. Alcuni esempi di applicazioni riguardano: (i) la simulazione degli effetti derivanti dalla percolazione di un contaminante in falda e l'analisi di possibili strategie di disinquinamento; (ii) per analisi da inserire in progetti di valutazione di impatto ambientale; (iii) l'applicazione delle Direttive Europee sull'acqua, come la Direttiva Nitrati per quanto riguarda il rispetto dei limiti di legge sull'utilizzo di fertilizzanti in ambito rurale.

I codici integrati in FREEWAT per tali scopi permettono di accoppiare un modello di flusso con un modello di trasporto advettivo-dispersivo di una o più specie chimiche, in zona satura e insatura. È possibile anche simulare semplici processi di reazione chimica, quali adsorbimento e biodegradazione. La simulazione del trasporto di calore è inoltre possibile trattando la temperatura come una particolare specie chimica e definendo i parametri necessari in maniera coerente. Un'ulteriore applicazione riguarda la possibilità di simulare flussi viscosità- e densità-dipendenti e questo risulta particolarmente utile per studi relativi al fenomeno dell'intrusione salina, dove le variazioni di densità dovute alla salinità sono fondamentali, o alla progettazione di impianti geotermici a bassa entalpia.

Il trasporto di soluti in zona satura viene trattato in FREEWAT mediante l'applicazione del codice di simulazione MT3DMS (Zheng and Wang 1999). La simulazione di flussi viscosità- e/o densità-dipendenti viene invece eseguita applicando il codice SEAWAT (Langevin et al. 2007), che accoppia MODFLOW-2005 (Harbaugh 2005) ed MT3DMS e risolve l'equazione di flusso in termini di altezza idraulica equivalente, tenendo cioè in considerazione le differenze relative di viscosità e densità di due fluidi in contatto (di cui uno è l'acqua che scorre tra i pori dei sedimenti di cui è costituito il sistema acquifero) e aggiornando ad ogni iterazione la concentrazione del fluido simulato, ossia quello contenuto nell'acquifero.

Per quanto riguarda il trasporto di soluti attraverso la zona insatura, sono disponibili due approcci:

- nel primo approccio (USB; Unsaturated Solute Balance; Borsi 2017) viene effettuata una stima della quantità di contaminante che, rilasciato al piano campagna, percola attraverso la zona insatura guidato da un flusso verticale puramente advettivo e raggiunge la tavola d'acqua. Tale quantitativo viene poi utilizzato per definire una sorgente a concentrazione costante che può disperdersi nella zona satura, in base ai processi advettivo-dispersivi simulati mediante MT3DMS;

- il secondo approccio è basato sull'applicazione del codice MT3D-USGS (Bedekar et al. 2016), che consente di accoppiare l'equazione di flusso e quella di trasporto per simulare processi advettivo-dispersivi in zona insatura.
Quelle appena descritte sono solo alcune delle potenzialità della piattaforma FREEWAT, un software di simulazione che si propone come strumento a supporto di una gestione integrata della risorsa idrica sotterranea, tentando di coniugare le esigenze di chi i modelli li sviluppa e le necessità di chi invece li usa.

\section{REFERENCES}

Bedekar V, Morway ED, Langevin CD, Tonkin M (2016) MT3DUSGS version 1: A U.S. Geological Survey release of MT3DMS updated with new and expanded transport capabilities for use with MODFLOW. U.S. Geological Survey, Techniques and Methods 6-A53, $69 \mathrm{p}$.

De Filippis G, Borsi I, Foglia L, Cannata M, Velasco Mansilla V, Vasquez-Suñe E, Ghetta M, Rossetto R (2017) Software tools for sustainable water resources management: the GIS-integrated FREEWAT platform. Rend. Online Soc. Geol. It. 42:59-61. doi:10.3301/ROL.2017.14

Hanson RT, Boyce SE, Schmid W, Hughes JD, Mehl SW, Leake SA, Maddock T, Niswonger RG (2014) One-water hydrologic flow model (MODFLOW-OWHM) (No. 6-A51). U.S. Geological Survey, Techniques and Methods 6-A51.

Harbaugh AW (2005) MODFLOW-2005, The U.S. Geological Survey Modular Ground-Water Model - the Ground-Water Flow Process. U.S. Geological Survey, Techniques and Methods 6-A16, 253 p.

Langevin CD, Thorne DT Jr, Dausman AM, Sukop MC, Weixing G (2007) SEAWAT Version 4: A Computer Program for Simulation of Multi-Species Solute and Heat Transport. U.S. Geological Survey, Techniques and Methods Book 6, Chapter A22, 39 p.

Rossetto R, Borsi I, Foglia L (2015) FREEWAT: FREE and open source software tools for WATer resource management. Rendiconti Online Società Geologica Italiana 35:252-255. doi: 10.3301/ ROL.2015.113

Schmid W, Hanson RT, Maddock III T, Leake SA (2006) User guide for the farm process (FMP1) for the US Geological Survey's modular three-dimensional finite-difference ground-water flow model, MODFLOW-2000. US Geological Survey, Techniques and Methods, 6-A17.

Schmid W, Hanson RT (2009) The Farm Process Version 2 (FMP2) for MODFLOW-2005-Modifications and Upgrades to FMP1 (No. 6-A32). US Geological Survey, Techniques and Methods 6-A32.

Zheng C, Wang PP (1999) MT3DMS, A modular three-dimensional multi-species transport model for simulation of advection, dispersion and chemical reactions of contaminants in groundwater systems; documentation and user's guide. U.S. Army Engineer Research and Development Center Contract Report SERDP-99-1, Vicksburg, MS, 202 p. 